Revue d'histoire de l'Amérique française

REVUE D'HISTOIRE DE L'AMÉRIQUE FRANÇAISE

\title{
L'enseignement universitaire catholique au Canada français de 1760 à 1860
}

\section{Léon Pouliot}

Volume 12, numéro 2, septembre 1958

URI : https://id.erudit.org/iderudit/301900ar

DOI : https://doi.org/10.7202/301900ar

Aller au sommaire du numéro

Éditeur(s)

Institut d'histoire de l'Amérique française

ISSN

0035-2357 (imprimé)

1492-1383 (numérique)

Découvrir la revue

Citer cet article

Pouliot, L. (1958). L'enseignement universitaire catholique au Canada français de 1760 à 1860. Revue d'histoire de l'Amérique française, 12(2), 155-169.

https://doi.org/10.7202/301900ar d'utilisation que vous pouvez consulter en ligne.

https://apropos.erudit.org/fr/usagers/politique-dutilisation/ 


\section{L'ENSEIGNEMENT UNIVERSITAIRE CATHOLIQUE AU CANADA FRANÇAIS DE 1760 À 1860}

Si, de 1760 à 1860 , il y a au Canada français un enseignement primaire et un enseignement secondaire, il n'y a pas d'enseignement universitaire, sauf pendant les toutes dernières années.

Comme celui-ci n'avait pas existé sous la domination française, il fallait le créer de toutes pièces, et la chose n'était pas facile. Indépendamment du problème financier, dont on ne saurait minimiser l'importance, deux conditions étaient simultanément requises pour que le Bas-Canada, de nationalité française, eût une institution de haut savoir. La première, c'était l'autorisation du pouvoir civil, sans laquelle les degrés académiques n'auraient eu aucune valeur; la seconde, c'était l'autorisation de l'Église. Car le Bas-Canada est catholique et la foi est le plus grand de tous les dons. L'Église a pour mission de la protéger chez ses enfants, de la défendre contre tout danger. Et hier comme aujourd'hui, aujourd'hui comme demain, les évêques ne peuvent collaborer à l'établissement d'une université, dès lors qu'ils sont convaincus qu'elle constituerait un grave danger pour la foi. Quand l'Église est impuissante à procurer à ses enfants les avantages d'une éducation universitaire catholique, elle permet la fréquentation des institutions non catholiques, et elle s'efforce d'en diminuer les dangers, en assignant, par exemple, des aumôniers aux étudiants. Mais de 1760 à 1860, cette formule d'apostolat n'est pas encore universellement établie dans l'Église; et au Canada on y était moins préparé qu'ailleurs. La date où les deux conditions simultanément requises sont vérifiées est 1852 ; et nous avons l'Université Laval.

Mais, ce n'était pas la première fois que l'on tentait de doter le Bas-Canada d'un enseignement universitaire. Et il nous paraît bon de rappeler, ne serait-ce que brièvement, quelques-uns des projets qui ont précédé Laval et qui méritent de retenir l'attention. 


\section{LE ROYALLES GEORGE COLLEGE}

Le premier, signalé par M. l'abbé Groulx, est de 1770 , soit dix ans après la conquête. ${ }^{1}$ Il est contenu dans une supplique adressée au gouverneur Carleton, au nom des habitants de Québec, des Trois-Rivières et de Montréal. Les signataires connaissent sans aucun doute l'existence du Séminaire de Québec. Ce qu'ils veulent, c'est autre chose; et bien que le mot université ne soit pas prononcé, la chose y est. Voyons plutôt.

Elever la jeunesse dans les bonnes mœurs, dans la probité, dans la vertu, dans l'étude des langues, dans les sciences de philosophie, de mathématiques, du génie, de la navigation, du droit civil, et généralement dans tous les arts et toutes les sciences humaines qui rendent l'homme utile à la société et qui font l'honneur d'une nation.2

Les moyens de réalisation sont faciles: il suffit de rendre à sa destination première le vaste collège des Jésuites de Québec, d'ajouter au personnel qui existe déjà dans le pays, six professeurs, tirés d'Europe «pour une fois seulement », d'appliquer au maintien et fonctionnement de l'institution les revenus des Biens des Jésuites. Les avantages sont justement soulignés. Ils attestent la hauteur de vue et la belle loyauté des auteurs de la supplique: empêcher le peuple de croupir dans l'ignorance, établir une juste égalité entre les anciens et les nouveaux habitants du pays, faire en sorte qu'il n'y ait désormais au Canada ni vaincus ni vainqueurs, mais uniquement des loyaux sujets de Sa Majesté. Et c'est le nom du roi que portera l'institution: le Royalles George College. Le projet fait honneur à ses auteurs. Mais il a le tort de venir trop tôt, c'est-à-dire à une heure où l'Angleterre n'a pas encore renoncé au dessein d'assimiler ses nouveaux sujets. Et comme soixante-dix ans s'écouleront encore avant qu'elle y renonce, ${ }^{3}$ on voit quel sort est réservé à tout projet d'université catholique et française au Bas-Canada.

1 Vers l'Emancipation (Montréal, 1921), 250 et ss.

2 Ibid.

3 Poulett Thomson, chargé de mettre à exécution l'Union des Canadas, écrivait, le 9 mars 1840, à Lord Russell, ministre des colonies: « Le grand objet que doit avoir en vue le gouvernement de Sa Majesté et celui que je vise certainement moi-même est de faire cette province essentiellement 


\section{LES TROIS PROJETS DE 1790}

Le deuxième et le troisième projet peuvent être rappelés $a d$ modum unius, puisqu'ils sont nés en même temps, s'inspirent d'un même document et tendent au même but: le projet de Lord Dorchester et celui de Simon Sanguinet. ${ }^{4}$

Le 31 mai 1787, Lord Dorchester instituait une commission, présidée par le juge William Smith et qui avait pour but d'enquêter sur les moyens d'augmenter l'éducation. Longtemps endormie, la commission se réveillait deux ans plus tard, lors du passage à Québec de l'évêque anglican de Halifax, Charles Inglis. Entre les moyens proposés pour obtenir le but, il y a un projet d'université neutre, c'est-à-dire ouverte aux catholiques et aux protestants et dans laquelle, sous prétexte de respecter les croyances de chacun, il n'y aura pas de Faculté de théologie. ${ }^{5}$ Invité à se prononcer, Mgr Jean-François Hubert, évêque de Québec, refusa sa collaboration. ${ }^{6}$ Si les arguments qu'il apporte sont de valeur inégale, si quelques-uns même nous paraissent aujourd'hui puérils, il ne faut pas oublier que son attitude lui était dictée par des raisons d'ordre religieux et que, dans l'atmosphère de l'époque, il ne paraissait pas prudent de trop y appuyer. En ce projet d'université s'insérant d'ailleurs dans un contexte historique plus large, où l'on prétendait « éclairer » les Canadiens et bannir graduellement l'usage de leur langue, il était difficile de ne pas y voir

anglaise. 》 Citation dans Groulx, Histoire du Canada français, III : 262. Russell lui-même n'était pas d'un autre avis. "On ne saurait donc reprocher à cette politique de manquer de clarté. Le propos avoué n'est pas seulement d'unir les deux provinces, mais de les unifier, et de les unifier, non pas dans le seul sens politique du mot, mais dans le sens national, culturel, de faire, en d'autres termes, que la partie anglaise assimile la partie française. 》 Ibid. 263.

${ }^{4}$ Louis-Philippe Audet, Le système scolaire de la Province de Québec (2 vol., Québec 1951), II: 141-219.

5 D'après Audet, op. cit., c'est Dorchester plus qu'Inglis qui tenait au caractère neutre de l'Université.

${ }_{6}$ Mand. des Evêques de Québec, II : 385-396, Lettre de Mgr Hubert en réponse au président du comité nommé pour l'exécution d'une université mixte en Canada. Québec, 18 novembre 1789. Ibid. 398-409, Mémoire de Mgr Bailly au sujet de l'Université, 5 avril 1790 . On sait que Mgr Bailly était favorable au projet d'université de Dorchester. Cette opposition entre le coadjuteur et l'évêque de Québec fit scandale à l'époque. Elle ne nous intéresse pas ici. 
un dessein d'assimilation. ${ }^{7}$ Quelques Canadiens français n'ont pas su le voir, à l'époque. Mais Mgr Hubert veillait. C'est à lui qu'il appartenait de décider s'il y avait, dans la condition générale de l'Église du Canada et dans son clergé, des moyens capables d'enrayer le danger. Il ne l'a pas cru; et pour notre part, nous n'osons pas le blâmer. Bien plus, nous croyons qu'il a bien servi l'Église et l'État, en ne compromettant pas la naissance, à une date ultérieure, d'une université catholique.

S'inspirait également par le rapport de la commission Smith, l'université rêvée par le riche avocat de Montréal, Simon Sanguinet, et en faveur de laquelle il faisait son testament en mars 1790. Quelques mois plus tard, une requête portant 175 signatures, parmi lesquelles celles de Mgr Bailly de Messein, coadjuteur de Québec, de M. Edmund Burke, directeur du Séminaire de Québec et futur Vicaire-Apostolique de Halifax, du R.P. Félix Bérey, Supérieur des Récollets de Montréal, demandait au gouverneur de donner suite au projet et de compléter la dotation, s'il le fallait, à même les revenus des Biens des Jésuites. ${ }^{8}$ Dorchester, en effet, recommanda le projet, mais en vain. Londres, semble-t-il, voulait laisser au futur régime parlementaire (1791) le soin d'apporter une solution au problème.

7 Inglis à Grenville, ministre des colonies, 8 septembre 1790: « Il n'y a peut-être pas un Canadien sur 500 qui comprenne l'anglais; et il est souhaitable, à tous les points de vue, qu'ils acquièrent la connaissance de cette langue, car cela contribuera à éclairer leurs esprits et en fera de meilleurs sujets. Règle générale, ils sont très ignorants et pas moins bigots, gardant d'étroits sentiments non libéraux (narrow illiberal sentiments) et cet attachement aveugle à la Papauté qui prévalait dans les basses classes en France lorsque le Canada fut colonisé au début du $17^{\mathrm{e}}$ siécle. Il faudrait cependant être prudent afin d'éviter ces démarches qui donneraient un contre-coup violent à leurs préjugés. (...) Un collège à Québec, conçu selon un plan libéral, avec un président protestant et des professeurs protestants et catholiques assurera la diffusion de l'instruction parmi les Canadiens ». Et Inglis y va de ses suggestions: après une période de quinze ou vingt ans, toutes les lois et documents officiels ne devraient être rédigés qu'en anglais et tous les fonctionnaires devraient connaître parfaitement cette langue. Audet, op. cit., 188-190.

8 Sur ce sujet, voir l'étude de M. Françis-J. Audet: \& Simon Sanguinet et le projet d'Université de $1790 »$, dans Mém. Soc. Roy. du Canada, 1936, sect. I : 53-70. 
La même année, 1790, le Séminaire de Montréal entre en scène, et dans la lettre qu'il adresse à Dorchester, au cours de l'été, il est dit:

Les Ecclésiastiques, désirant seconder les intentions favorables de Votre Excellence pour encourager l'éducation, ils ne croient pouvoir mieux répondre à la destination des biens qu'ils possèdent que de fonder un collège dans la ville de Montréal. En conséquence de quoi, ils demandent «une charte ou Lettres patentes portant permission aux ecclésiastiques du Séminaire de Montréal d'acquérir les terrains et emplacements situés en la dite ville de Montréal entre la place d'Armes, la rue St-Jacques, la rue St-François et la rue Notre-Dame, pour y fonder un collège, sous le nom de Dorchester, où l'on enseignera à lire et à écrire les langues anglaise, française et latine, les Belles-Lettres, la Philosophie, les mathématiques, le droit civil et les autres sciences qui seront jugées nécessaires, sous la direction de l'évêque de Québec ou diocésain et des Supérieurs et Directeurs du dit Séminaire avec droit d'en choisir et nommer les maîtres et professeurs parmi les sujets de Sa Majesté Britannique et de faire les règlements nécessaires pour la régie intérieure du dit Collège... 9

"En somme, conclut Mgr Maurault, comme il appert par la proposition d'enseigner le droit civil et les autres sciences qui seront jugées nécessaires, le Séminaire veut fonder à Montréal une université pour faire pièce à l'université neutre demandée par la Commission d'éducation. ${ }^{10}$ Appuyée par une pétition portant 89 signatures, la demande du Séminaire fut présentée au Conseil Exécutif, qui résolut de la déférer à $\mathrm{Sa}$ Majesté, et les choses en restèrent là.

\section{LE PROJET DES ÉVÊQUES (1843-1846)}

C'est en 1843, croyons-nous, qu'il faut placer le premier geste des évêques relatif à la fondation d'une université catholique dans le Bas-Canada. Au mois de mai, Mgr Bourget apprend que le ministère LaFontaine-Baldwin se propose d'assigner, à la prochaine session, une fin particulière aux Biens des Jésuites et que

${ }^{9} \mathrm{Mgr}$ Olivier Maurault, p.s.s. Le Petit Séminaire de Montréal (Montréal 1918), 31-32.

10 Ibid. 32. 
le clergé catholique aura l'occasion de se faire entendre. En communiquant cette nouvelle à l'évêque de Québec, il suggère de réclamer ces Biens pour l'Église du Bas-Canada. ${ }^{11}$ Aussitôt dit aussitôt fait, et avant la fin de mai une requête dans ce sens est adressée au gouverneur Metcalfe. Elle porte les signatures de Mgr Signay, évêque de Québec, de Mgr Bourget, évêque de Montréal et de Mgr Turgeon, coadjuteur de Québec. ${ }^{12}$

Le 30 juin, l'évêque de Montréal raconte l'entretien qu'il a eu avec Louis-Hippolyte LaFontaine relative aux Biens des Jésuites. Le Procureur général a paru surpris et décontenancé en apprenant que les évêques avaient adressé une requête à Metcalfe,

parce qu'il craint qu'elle n'entrave leurs mesures pour se mettre en possession de ces riches propriétés. Il m'a avoué qu'il y avait $£ 20,000$ dans cette caisse; et que leur projet était par dessus tout de faire restituer ces biens en faveur de l'éducation dans le Bas-Canada. Il ne m'a pas caché que les protestants auraient leur part à ce gâteau. Il serait, je crois, assez d'opinion d'en appliquer une partie à la dotation des collèges de l'ancienne Province du Bas-Canada 13

Jusqu'ici nous savons que les évêques du Bas-Canada réclament les Biens des Jésuites. Mais à quelle fin ? C'est M. Hyacinthe Hudon, vicaire général de Montréal, qui nous l'apprend. Chargé de surveiller les intérêts de l'Église au Parlement de Kingston, il écrit à Mgr Turgeon, le 14 octobre 1843:

Afin de demander aux membres du ministère d'abandonner le plan dont Mgr [Bourget] vous a envoyé copie, il faudrait au moins pouvoir leur en offrir un autre qui pût remplacer celui-là, et pourvoir à l'établissement d'une université, puisque nos membres tiennent tant à une université. J'ai donc pris la liberté de vous envoyer un projet fait à la hâte, et qui n'a pas eu le temps d'être beaucoup mûri, priant V.G. d'y faire tel changement, amendement, etc. qu'elle jugera à propos, ou d'en suggérer un autre, mettant celui-ci totalement de côté. ${ }^{14}$

11 AAM (Archives archevêché de Montréal) Lettres de Mgr Bourget, III : 75-76. A Mgr Signay, 11 mai 1843.

12 AAM : Pièces et Actes, IV: f. 226v-227v. A Sir Charles Metcalfe: Requête des Evêques du ci-devant B. Canada pour demander que les Biens des Jésuites soient rendus à L'Eglise catholique.

13 A AM. Lettres de Mgr Bourget, III : 110-120. A Mgr Signay.

14 AAM. Lettres de Mgr Bourget, III : 201-202. 


\section{Et Mgr Bourget à Mgr Turgeon :}

Je prends la liberté de vous envoyer un projet de Bill pour l'établissement de l'Université de Toronto, afin que V.G. puisse voir et s'assurer que les principes professés dans l'écrit anonyme qui nous a été communiqué sont mis à exécution: et que si on veut nous imposer une université à Québec, nous devons nous attendre à être mis sur le même pied. ${ }^{15}$

Il résulte de ces documents: qu'à l'occasion d'un projet de loi relatif à l'Université de Toronto, les ministres du Bas-Canada veulent établir une institution de haut savoir à Québec; qu'il y a au moins coïncidence entre ce projet et l'appropriation que le ministère entend faire des Biens des Jésuites; que, dans la pensée de LaFontaine, cette appropriation sera toute à l'avantage de l'éducation dans le Bas-Canada et que les protestants y auront leur part.

Il n'est pas sûr que les évêques aient l'intention de fonder une université catholique, au moment où ils réclament les Biens des Jésuites; mais cette intention apparaît clairement dans la lettre de M. Hudon, qui est du mois d'octobre. Quant aux ministres, tout nous invite à croire qu'ils y pensaient, eux, dès le début et qu'ils n'étaient pas favorables à l'établissement à Québec d'une université qui serait régie par d'autres principes que celle de Toronto. Avons-nous tort de croire que le projet caressé par les ministres de 1843 ressemble comme un frère au projet de Dorchester, et qu'il s'agissait encore d'une université neutre ? N'est-ce pas pour cette raison que les évêques veulent le remplacer par un autre?

Quoiqu'il en soit, il ne semble pas que Metcalfe ait répondu à la requête des évêques du Bas-Canada. Les difficultés du gouverneur avec la Chambre d'Assemblée, la démission du ministère et la longue vacance qui suivit, eurent pour effet de différer le projet.

Les évêques profitent de ce délai pour refaire leur unité ${ }^{16}$

15 Ibid. 210-211. 21 octobre 1843.

$16 \mathrm{Mgr}$ Power, évêque de Toronto, avait protesté contre la requête des évêques du Bas-Canada à Metcalfe. Il réclamait une part des Biens des Jésuites pour les catholiques du Haut-Canada. 
et pour mûrir leur dessein. Une nouvelle requête est adressée à Metcalfe, signée celle-là, par tous les Ordinaires du Haut et du Bas-Canada. ${ }^{17}$ Elle réclame les Biens des Jésuites pour l'Église du Canada et le projet d'université est exprimé en toutes lettres:

Vos pétitionnaires n'hésitent pas à prendre l'engagement solennel de fonder, à même les revenus de ces biens, un ou plusieurs établissements d'éducation supérieure dans lesquels seront enseignées telles branches de sciences que pourraient requérir les besoins du pays, et même d'y établir des cours de médecine, de droit, d'économie domestique et même des beaux-arts, adaptés aux besoins de toutes les classes de la société, dès qu'il se présenterait un nombre suffisant d'élèves pour suivre ces cours; et pour cela un projet d'éducation est aussi annexé au mémoire susmentionné.18

C'est dans ce Projet d'Éducation que s'affirment la pensée et la volonté des évêques :

La conservation de la foi catholique dans le Canada se trouve garantie par l'acte fondamental de l'incorporation de cette Colonie à la nation anglaise, et par la promesse solennelle des Souverains. Ses droits sont donc consacrés, et pour qu'ils ne soient pas un vain nom, ils doivent exercer une influence salutaire sur toutes les classes de la société et à tous les âges de la vie ... La population catholique du Canada, dans l'intérêt de sa foi, et pour laisser intacte à ses enfants cette part la plus belle de l'héritage de ses pères, sous la protection d'un gouvernement plein de bienveillance et de justice, a donc le droit de désirer un enseignement complet, qui convienne aux classes industrielles et commerciales, et qui satisfasse en même temps aux besoins des classes élevées de la société et des hommes de profession, avec toutes les garanties d'orthodoxie et de moralité que demandent les intérêts de la foi...

Les universités protestantes peuvent suffire à la population de cette croyance; elles ne peuvent convenir à la population catholique sans de graves inconvénients pour sa foi catholique....

17 AAM. Pièces et Actes, V: ff. 121-122. Janvier 1845. Signataires de la requête: Mgr Signay (Québec), Mgr Gaulin (Kingston), Mgr Bourget (Montréal), Mgr Power (Toronto), Mgr Turgeon (coadj. de Québec), Mgr Phelan (coadj. de Kingston), Mgr Prince (coadj. de Montréal).

18 Citation dans Paul Desjardins, s.j., Le Collège Sainte-Marie de Montréal (2 vol., Montréal, 1944), II : 46. 
Si l'influence de l'Autorité ecclésiastique se borne à un rôle secondaire ou consultatif; si elle n'a droit qu'à de simples avis ou à une simple inspection; si son enseignement se borne à quelques leçons orales, sur le rang des leçons de mathématiques ou de langues, son intervention est inutile et plus souvent inefficace; elle sera toujours insuffisante et incomplète.

Disons donc que l'autorité religieuse, pour remplir sa mission, doit s'exercer sur le choix des maîtres, le cadre de l'enseignement et les mesures réglementaires; sans cela, le principe de l'éducation religieuse n'est qu'un nom, on a exclu la chose....

Le dessein des Évêques Catholiques du Canada serait donc de fonder une université où l'on pourrait prendre les degrés qui permettent d'exercer les professions libérales. 19

Voilà qui est net et franc ! Enfin, preuve que les évêques avaient mûri le projet, une distribution des études était proposée, tant au stade collégial qu'au niveau universitaire. ${ }^{20}$

Quelle fut l'attitude de Metcalfe devant cette seconde instance des évêques ? Nous ne le savons pas. Mais Mgr Bourget re-

19 Ibid: 52-53.

20 Ibid: 54: Distribution des études du collège: 1. Cours communs aux deux sections qui le composent: les études religieuses - la langue et la littérature française et anglaise - la philosophie - l'histoire ancienne, moderne, nationale - les éléments de physique et de chimie — les éléments d'histoire naturelle (zoologie, botanique, minéralogie, géologie) - les mathématiques, arithmétique, algèbre, géométrie, trigonométrie - les arts d'agrément, dessin, peinture, musique, gymnastique.

2. Cours spéciaux pour le commerce et l'industrie: Mathématiques commerciales, tenue des livres - économie politique et industrielle - Mécanique, histoire, composition et usage des machines - mathématiques développées, géométrie analytique et descriptive - application des mathématiques à la perspective, à l'architecture civile et militaire, au dessin des machines, à la coupe des pierres, à la charpente-physique et chimie industrielle, agriculture, arpentage - dessin linéaire, topographique, architectural, dessin d'ornement, modelage.

3. Cours spéciaux pour les cours universitaires: les langues et la littérature grecque et latine.

ETUDES SUPERIEURES DE L'UNIVERSITE:

Le droit - la médecine - les Belles-Lettres — les sciences naturelles, historiques, philosophiques, mathématiques.

Les dépenses à faire auraient pour objet: 1) la construction et l'entretien d'un édifice convenable; 2) l'ameublement, les livres, les collections; 3) l'entretien des professeurs; 4) la fondation d'un certain nombre de bourses à mettre en concours pour exciter l'émulation; 5) les prix et récompenses. 
vient à la charge auprès de son successeur, Cathcart. Le 9 février 1846, il lui adresse une copie de la requête et il demande une audience. ${ }^{21}$ Le 30 mars, il écrit à Denis-Benjamin Viger, chef avec Draper du Conseil Exécutif :

Les catholiques doivent désirer être sur le même pied que leurs frères co-sujets britanniques. Or, ceux-ci ayant à Toronto une université richement dotée, les catholiques ont un droit spécial de réclamer le même avantage pour eux. Le gouvernement n'aurait aucun frais à supporter pour la fondation d'une université catholique; car il suffirait pour cela de laisser les biens du ci-devant Ordre des Jésuites à la disposition des évêques, qui osent répondre qu'ils satisferont aux besoins du pays et qu'ils atteindront la fin que l'on doit se proposer dans une Institution de ce genre. ${ }^{22}$

On peut admirer cette belle audace et cette belle assurance. On se rend compte, après cent ans, qu'elles étaient téméraires. En admettant que les $£ 20,000$ annuels des Biens des Jésuites soient l'équivalent de $£ 200,000$ d'aujourd'hui, il n'est pas sûr qu'ils auraient suffi à la construction des édifices, à leur entretien, à la formation et au salaire des professeurs ainsi qu'aux autres frais d'administration. C'est plutôt le contraire qui est certain, et pas n'est besoin de le démontrer.

Le 26 mai 1846, la Chambre d'Assemblée refusait de se rendre au désir des évêques et décidait que les revenus des Biens des Jésuites seraient appliqués à l'éducation tant des catholiques que des protestants du Bas-Canada.

Les évêques s'empressent de demander à Cathcart de ne pas sanctionner le bill. Démarche inutile, car le 9 juin le bill recevait la sanction de Son Excellence.

Est-ce la fin ? Pas encore. Et c'est la ténacité de Mgr Bourget, croyons-nous, qu'il faut voir dans la Requête du clergé catholique des diocèses de Québec et de Montréal au sujet des Biens des Jésuites. ${ }^{23}$ La réponse du nouveau gouverneur, Elgin, ne

21 AAM. Lettres de Mgr Bourget, IV: 89.

22 AAM. Lettres de Mgr Bourget, IV: 109.

${ }_{23}$ Mand. des Evêques de Québec, III: 514-515. Juin 1847; « Vos pétitionnaires, considérant que la Législature, dans sa dernière session, n'a approprié, que pour un an seulement, le revenu de ces biens, dans le but, sans doute, de mieux connaître les intentions du clergé et du peuple catholique 
laissait aucune place à l'espoir. ${ }^{24}$ "Espérons, écrit Mgr Bourget, que la Divine Providence, après nous avoir fait passer ce mauvais temps, nous fera arriver à des jours plus sereins, et qu'elle suscitera des hommes justes, qui rendront à chacun ce qui lui appartient $\gg^{25}$

\section{LE PROJET DE SAINT.HYACINTHE (1848-1849)}

L'évêque de Montréal n'était pas le seul à espérer contre toute espérance. Et c'est ici qu'il faut rappeler le projet, plus poétique que réaliste, raconté par Mgr Choquette dans son Histoire du Séminaire de Saint-Hyacinthe. ${ }^{26}$

Or donc, le 19 juillet de l'an de grâce 1848 , le gouverneur général, Lord Elgin, était reçu au Séminaire de Saint-Hyacinthe. C'était la fin de l'année scolaire: un véritable régal de l'esprit, qui se prolongeait parfois jusqu'à trois jours pleins et qui faisait salle comble. Et pourquoi pas ? Des élèves dûment stylés, qui savent se présenter, exposent, discutent et règlent les problèmes les plus divers, depuis la grammaire et l'histoire jusqu'à la métaphysique, sans oublier en cours de route la physique et la chimie. Le noble lord en fut émerveillé, sidéré. Il le disait à qui voulait l'entendre, et surtout aux directeurs du Séminaire.

Un tel témoignage, et venu de si haut, n'était-ce pas comme un ordre, ou du moins comme un signe qu'il fallait aller de l'avant ? Et voilà que, pendant les vacances qui suivent, les directeurs se prennent à rêver. Pourquoi le Séminaire de Saint-Hya-

de cette province, croient devoir représenter à Votre Excellence que toute appropriation de ces biens qui tendrait à les divertir de leur destination première, serait pour eux un sujet d'affliction profonde ».

24 Ibid. 516. D. Daly, secrétaire provincial, à M. C.-F. Cazeau, secrétaire de l'archevêché de Québec: * Son Excellence m'enjoint de vous faire remarquer que la Législature a formellement approprié les revenus des biens des jésuites aux fins de l'éducation, et que ces revenus par conséquent n'en peuvent être détournés pour être remis entre les mains du clergé de l'Eglise de Rome, sans la sanction préalable de la Couronne et de la Législature, et Son Excellence est avisée que c'est un objet qu'il n'est ni expédient ni désirable de chercher à obtenir.

Dans ces circonstances, Son Excellence se trouve dans l'impossibilité de prendre aucune mesure à l'effet d'accomplir le désir des requérants ». Juin 1847.

25 AAM. Lettres de Mgr Bourget, IV: 292. A M. C.-F. Cazeau, 28 juillet 1847.

26 I : 292-293. 
cinthe ne serait-il pas le siège de la future université catholique? «Bâtissez, avait dit Georges-Étienne Cartier, nous vous donnerons cent mille dollars. » Et puis, poursuit Mgr Choquette:

Le site même désignait Saint-Hyacinthe comme le lieu le plus propre à l'établissement d'une université. Une petite ville de campagne, reliée à la métropole par un chemin de fer qui s'achevait, c'était Oxford par rapport à Londres. En fallait-il davantage pour susciter dans l'esprit des directeurs et de leurs amis les plus chimériques espoirs.

Et le rêve se continue; on a l'impression qu'il va bientôt devenir réalité.

Le 10 février 1849, avait lieu l'inauguration du GrandTronc. Le sympathique Lord Elgin reparaît à Saint-Hyacinthe, et il n'est pas seul. Les ministres, les députés, le maire et les échevins de Montréal ont voulu participer à cet événement historique; en tout quelque 150 personnes et toutes personnes de qualité. La délégation, il va sans dire, fut reçue au Séminaire; occasion unique pour rendre notoire le projet cher aux directeurs. Et dans l'adresse que l'élève Michel Godard lit à Son Excellence, si le mot université n'est pas prononcé, la chose y est:

\footnotetext{
Cette communication que Saint-Hyacinthe voit s'ouvrir avec Montréal, avec les townships de l'Est et les Etats-Unis, est de la plus haute importance pour cette Institution. Le nombre des élèves doit nécessairement s'augmenter, et la position même de cette maison qui la fera visiter par tant de voyageurs, y amènera un plus large développement des études dans l'intérêt de l'honneur national. C'est donc un avenir plein de grandeur qui s'ouvre aujourd'hui pour ce collège. Cette destinée nouvelle s'inaugure aujourd'hui par l'honorable visite qu'il reçoit. C'est un présage de sa grandeur future. En attendant ces jours heureux....27
}

Ne soyons pas étonnés après cela, si les projets d'agrandissement, déjà à l'étude, reçoivent une impulsion nouvelle, et si la conception des plans est inspirée par des préoccupations universitaires. Ces plans - une miniature en bois - on avait suggéré aux directeurs de les exposer au Parlement de Montréal comme argument à l'appui d'une demande d'allocation. La maquette fit,

\section{Ibid.}


non sans peine, le voyage de Saint-Hyacinthe à Montréal, où elle fut irrémédiablement perdue dans l'incendie du Parlement.

Ici finit le projet de Saint-Hyacinthe; mais il n'est pas inutile. Il nous apprend qu'en 1848-1849, un groupe d'hommes qui comptent parmi les plus éclairés de l'époque, pédagogues de carrière, donnés tout entiers à la formation de la jeunesse, fortement attachés à la vraie grandeur et à la vraie gloire de la patrie, éprouvaient le besoin d'une institution de haut savoir; et ils en croyaient la réalisation possible sans le secours, jugé jusque-là indispensable des Biens des Jésuites. Il y a là quelque chose de nouveau et quelque chose de grand. Il importait de le signaler.

\section{VERS L'UNIVERSITÉ LAVAL}

Pendant les années qui suivent immédiatement cet échec, si des individus s'intéressent encore au projet d'université catholique, les évêques semblent l'avoir oublié. Du 1er au 11 mai 1850, ils se réunissent à Montréal, sous la présidence de Mgr Turgeon, administrateur du diocèse de Québec. Les résultats de leurs délibérations nous sont connus par une circulaire au clergé ${ }^{28}$ et par une Pastorale aux fidèles ; ${ }^{29}$ il n'y est pas question d'enseignement universitaire.

A cette même assemblée, ils ont fixé au 15 août 1851 la réunion du premier Concile provincial de Québec. C'est dire que les lettres qui s'échangent entre Québec et Montréal sont plus fréquentes que jamais. Mgr Turgeon, ayant consulté Mgr Bourget sur l'opportunité d'étudier au Concile certains problèmes relatifs aux collèges, l'évêque de Montréal répond aux questions précises du Métropolitain et il ajoute:

A propos de Séminaires et de collèges, V.G. croirait-elle que le Concile devrait s'occuper de la fondation d'une Université et de l'érection régulière de collèges dépendant de cette Maison-Mère d'éducation? Ne serait-il pas temps de prendre les devants et de nous faire constituer légalement maîtres de l'enseignement catholique ? Pourrait-on refuser au BasCanada ce qu'on a accordé au Haut? Le Gouvernement ne serait-il pas forcé par la nécessité des choses de nous accor-

28 Mand. des évêques de Montréal, II : 115-126.

29 Ibid. VIII : 64-86. 
der les privilèges ordinaires aux Universités lorsqu'on ne lui demanderait aucuns fonds provinciaux pour la dotation de notre Institution qui nous mettrait sur le pied d'égalité avec McGill University et Queen's College? La crainte que les laïques ne s'emparent plus tard ici, comme en France, de l'éducation ne serait-elle pas une raison pressante pour nous de travailler du moins à réaliser ce projet. Ne serait-elle pas aussi pour les Séminaires et collèges un urgent motif de s'entendre à l'amiable entre eux pour n'être pas exposé au monopole universitaire ? N'est-il pas vrai que le Séminaire de Québec serait, plaudentibus omnibus, choisi pour être l'Université catholique de notre Amérique Britannique? Quelle force nous aurions dans une pareille institution, après que nous aurions obtenu, comme de droit, la sanction pontificale ! C'est une idée comme une autre que j'envoie à la bonne aventure. Elle fera ou ne fera pas son chemin, c'est autre chose. Je la dépose, pour ce qu'elle vaut, aux pieds de Votre Grandeur. 30

Cette idée, que Mgr Bourget envoyait à la bonne aventure, allait avoir des conséquences qu'il n'avait pas prévues. Mais, pour le moment, tout va pour le mieux dans le meilleur des mondes. Mis au courant par Mgr Turgeon de la suggestion de Montréal, le Conseil du Séminaire de Québec, répondait, le 4 avril 1851, que "Nos Seigneurs les Évêques le trouveront toujours disposé à faire ce que pourra exiger le bien de la religion et du pays. ${ }^{31}$

Dans une lettre subséquente, Mgr Bourget propose que le Concile revise tout notre système d'éducation, afin de le mieux adapter aux besoins du pays.32 La suggestion n'était pas sans mérite; mais elle venait trop tard. Mgr Turgeon n'y donna pas suite, et il eut raison, croyons-nous. Les évêques n'avaient pas le temps de se renseigner sur tant de questions fort délicates: car chaque collège avait son programme, ses méthodes d'enseignement, ses traditions, ses problèmes financiers, ses prétentions, peut-être ... Et puis, le Concile, comme tel, avait-il compétence pour faire droit aux suggestions de Mgr Bourget, si opportunes qu'elles fussent par ailleurs?

Le Concile se contenta donc de déclarer, dans son $\mathrm{XV}^{\mathrm{e}}$ décret,

30 AAM. Lettres de Mgr Bourget, VI : 395-401.

31 Registre des délibérations du Séminaire de Québec.

32 AAM. Lettres de Mgr Bourget, VI: 408-411. 
que rien ne serait omis pour que les catholiques aient leurs écoles, leurs collèges, leurs universités. ${ }^{33}$

Entre-temps, le Séminaire de Québec s'est donné un nouveau Supérieur, dans la personne de M. Louis-Jacques Casault. Et les choses vont bon train, plus vite que ne l'avait prévu $\mathrm{Mgr}$ Bourget. Mais c'est là une histoire que nous n'avons pas à raconter ici. Il nous suffit de dire quel était l'enseignement universitaire catholique au Canada français en 1860, terme de notre enquête.

Le 8 décembre 1852, Londres accordait au Séminaire de Québec une charte universitaire impériale. L'indult de Rome, permettant au Grand Séminaire de Québec de conférer les degrés ecclésiastiques à ses élèves, est du 6 mars 1853. Les Facultés de médecine et de droit sont instituées dans le plus court délai possible. En 1860, elles ont déjà conféré et elles continuent de conférer les degrés académiques. Les deux autres Facultés, Théologie et Arts, sont en pleine voie d'organisation. Rien n'a été négligé pour faire de l'Université Laval une institution de haut savoir vraiment digne de ce nom: formation et voyages des professeurs à l'étranger, construction d'un pensionnat, développement des bibliothèques, des musées, des laboratoires.

L'institution est à la charge du Séminaire, propriétaire de la charte, et, en 1862, elle lui a déjà coûté la somme fabuleuse pour l'époque de $\$ 296,000$. Ce n'est pas tout; car le dernier exercice financier se solde par un déficit de $\$ 11,000 .^{34}$

Tout cela ne s'est pas fait sans heurts. Mais l'essentiel, c'est qu'en 1860 l'enseignement universitaire catholique existe au Canada français, qu'il est venu pour rester et grandir.

Il est juste de reconnaître la haute conception qu'en avaient les directeurs du Séminaire de Québec ainsi que les généreux sacrifices qu'ils ont consentis pour en doter le pays; juste aussi de remercier tous ceux qui, à des degrés divers, ont contribué au succès.

LÉON POULIOT, s.j.

33 Nobis vero nihil non emoliendum erit ut catholici jura sua retinentes, scholis propriis sicut et collegiis, universitatibusque, in tota nostra provincia fruantur ».

${ }^{34}$ Mémoire de l'Université Laval (Québec, 1862). 